\title{
Crystal Structures of Complexes of Bacterial DD-Peptidases with Peptidoglycan-Mimetic Ligands: The Substrate Specificity Puzzle
}

\author{
Eric Sauvage ${ }^{1}$, Ailsa J. Powell ${ }^{2}$, Jason Heilemann ${ }^{2}$, \\ Helen R. Josephine ${ }^{3}$, Paulette Charlier $^{1}$, Christopher Davies ${ }^{2}$ \\ and R. F. Pratt ${ }^{3 *}$
}

${ }^{1}$ Centre d'Ingéniere des

Proteines, Université de Liège, B-4000 Sart Tilman, Liège, Belgium

${ }^{2}$ Department of Biochemistry, Medical University of South Carolina, Charleston, SC 29425, USA

${ }^{3}$ Department of Chemistry, Wesleyan University, Middletown, CT 06459, USA

Received 12 March 2008; received in revised form 28 May 2008; accepted 1 June 2008 Available online 10 June 2008
The X-ray crystal structures of covalent complexes of the Actinomadura R39 DD-peptidase and Escherichia coli penicillin-binding protein (PBP) 5 with $\beta$ lactams bearing peptidoglycan-mimetic side chains have been determined. The structure of the hydrolysis product of an analogous peptide bound noncovalently to the former enzyme has also been obtained. The R39 DDpeptidase structures reveal the presence of a specific binding site for the D- $\alpha$ aminopimelyl side chain, characteristic of the stem peptide of Actinomadura R39. This binding site features a hydrophobic cleft for the pimelyl methylene groups and strong hydrogen bonding to the polar terminus. Both of these active site elements are provided by amino acid side chains from two separate domains of the protein. In contrast, no clear electron density corresponding to the terminus of the peptidoglycan-mimetic side chains is present when these $\beta$-lactams are covalently bound to PBP5. There is, therefore, no indication of a specific side-chain binding site in this enzyme. These results are in agreement with those from kinetics studies published earlier and support the general prediction made at the time of a direct correlation between kinetics and structural evidence. The essential highmolecular-mass PBPs have demonstrated, to date, no specific reactivity with peptidoglycan-mimetic peptide substrates and $\beta$-lactam inhibitors and, thus, probably do not possess a specific substrate-binding site of the type demonstrated here with the R39 DD-peptidase. This striking deficiency may represent a sophisticated defense mechanism against low-molecular-mass substrate-analogue inhibitors/antibiotics; its discovery should focus new inhibitor design.

(C) 2008 Elsevier Ltd. All rights reserved.

Edited by G. Schulz

Keywords: penicillin-binding proteins; DD-peptidases; $\beta$-lactam; peptidoglycan; peptide

\section{Introduction}

Cell wall growth is integral to bacterial proliferation. The bacterial cell wall is composed of peptidoglycan and grows by incorporation of disaccharidepentapeptide monomers. This occurs outside the cell membrane and requires two kinds of enzyme active site that separately catalyze transglycosylation and transpeptidation reactions. ${ }^{1}$ The latter reaction serves

${ }^{*}$ Corresponding author. E-mail address: rpratt@wesleyan.edu.

Abbreviation used: PBP, penicillin-binding protein. to stabilize the cell wall by crosslinking the glycan strands. It is catalyzed by a class of enzymes known as D-alanyl-D-alanine transpeptidases (since they catalyze the reaction of Scheme 1), or otherwise simply as DD-peptidases, or, inasmuch as they are the targets of $\beta$-lactam antibiotics, as penicillin-binding proteins (PBPs).

As shown in Scheme 1, the DD-peptidases employ an acyl-(serine)enzyme intermediate that can either be aminolyzed (by $\mathrm{R}^{\prime} \mathrm{NH}_{2}$ ) to complete the peptidoglycan crosslink or be hydrolyzed in a carboxypeptidase reaction to limit the extent of crosslinking. It seems now quite well established that the essential crosslinking reaction is catalyzed by a high-mole- 


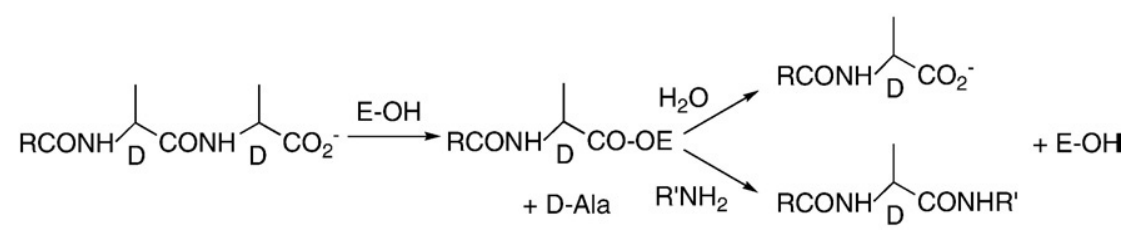

Scheme 1. The transpeptidase and carboxypeptidase reactions catalyzed by a DD-peptidase.

cular-mass (60-100 kDa) group of DD-peptidases, while a lower-molecular-mass (40-60 kDa) group appears to have in vivo only DD-carboxypeptidase and DD-endopeptidase activities. ${ }^{2,3}$ In vitro, however, several members of the latter group are also able to catalyze transpeptidation reactions with small peptides and esters. ${ }^{3,4}$ The enzymatic activity of these enzymes has recently been reviewed.

In view of their in vivo role as DD-peptidases, one would anticipate that these enzymes would exhibit substrate specificity towards peptides that are analogous in structure to oligopeptide elements of peptidoglycan. In certain instances, this appears to be correct. For example, peptide 1 is a very specific substrate of the low-molecular-mass DD-peptidase of Streptomyces R61, which catalyzes both transpeptidase and carboxypeptidase reactions with this substrate as acyl donor. ${ }^{6-8}$ In view of Streptomyces peptidoglycan structure (peptide 2), this result is logical, although it is striking that peptide 3 , containing another significant element of peptide 2 , is a poor substrate comparable to generic D-alanyl-D-<smiles>CC(CCCCC(=O)NC(C)C(=O)NC(C)C(=O)[O-])NC(=O)C[NH3+]</smiles><smiles>[2H]C(CCC(=O)NC(C)(C)C(=O)NC(C)C(=O)NC(C)C(=O)NC(C)C(=O)O)NC(=O)C(C)NC(C)=O</smiles><smiles>[M]OC(C)C(=O)NC(C)C(=O)NC([2H])CCC(=O)NC(C)(CCCC(C)NC(=O)C[NH3+])C(=O)NC(C)C(=O)NC(C)C(=O)NC(C)C(=O)O[Na]</smiles><smiles>CC(CCCCC(=O)N[C@H]1C(=O)N2C(C(=O)[O-])C(C)(C)S[C@H]12)NC(=O)C[NH3+]</smiles><smiles>CC1(C)S[C@@H]2[C@H](NC(=O)CCCCC([NH3+])C(=O)[O-])C(=O)N2C1C(=O)[O-]</smiles><smiles>CC(=O)OCC1=C(C(=O)[O-])N2C(=O)[C@@H](NC(=O)CCCCC([NH3+])C(=O)[O-])[C@H]2SC1</smiles><smiles>CC(NC(=O)C(C)NC(=O)CCCCC([NH3+])[O+]([O-])[O-])C(=O)[O-]</smiles> 
alanine-terminating peptides. ${ }^{9}$ As would be expected on the basis of these results, crystal structures show that peptide 1 binds very snugly to the R61 DDpeptidase active site, which contains what appears to be a specific binding site for the glycyl-L-aminopimelyl moiety. ${ }^{10} \beta$-Lactams with this same side chain (e.g., peptide 4) are also extremely potent covalent inhibitors of the R61 enzyme and employ the same specific side-chain binding site. ${ }^{11,12}$

A survey of examples of the major classes of bacterial DD-peptidases, however, showed that the above result is not general, at least with respect to specificity in substrate turnover and $\beta$-lactam-inhibitory kinetics. ${ }^{9,13}$ The high-molecular-mass enzymes, for example, do not show any particular preference for peptidoglycan-mimetic peptides and $\beta$-lactams analogous to peptides 1 and $\mathbf{3}$ or $\beta$-lactam 4, nor do certain low-molecular-mass enzymes (e.g., Escherichia coli PBP5 and Streptococcus pneumoniae PBP3). ${ }^{9,13}$ On the basis of the R61 DD-peptidase result, we predicted that the crystal structures of enzymes that show kinetic specificity for peptidoglycanmimetic peptides and $\beta$-lactams will contain specific binding sites for the side chains of these molecules, whereas those that show no kinetic specificity will not. ${ }^{13}$ To support these predictions, we describe in this article the crystal structures of complexes of two lowmolecular-mass DD-peptidases with peptidoglycanmimetic ligands, the $\beta$-lactams 5 and 6 , and the peptide 7 bound to them. Kinetics studies showed that the Actinomadura R39 DD-peptidase does display peptidoglycan-mimetic substrate specificity analogous to that of the R61 enzyme, but E. coli PBP5 does not. ${ }^{9,13}$

The Actinomadura R39 DD-peptidase is a low-molecular-mass class $C$ enzyme ${ }^{2}$ with strong amino acid sequence similarity to E. coli PBP4 and Bacillus subtilis PBP4a. ${ }^{14,15}$ Crystal structures have confirmed the structural resemblance. ${ }^{16-18}$ The R39 DD-peptidase is a water-soluble enzyme that is loosely associated with the bacterial cell membrane. Although the precise role of the enzyme in vivo is not known, under in vitro conditions, it has been shown to catalyze the hydrolysis and aminolysis of small D-alanyl peptides and esters. $^{19,20}$

PBP5 of E. coli is also categorized as a low-molecular-mass PBP, but of class A. ${ }^{2}$ It is not essential to the survival of E. coli, but its absence affects cell morphology. ${ }^{21}$ Under normal conditions, it is apparently responsible for much of the D-alanine carboxypeptidase activity that limits cell wall crosslinking. ${ }^{22}$ In vivo, PBP5 is attached to the E. coli inner membrane by a C-terminal $\alpha$-helix, ${ }^{23}$ removal of which affords the solubilized protein used for kinetics studies and crystal structure determination. ${ }^{24}$ The solubilized construct is inhibited by $\beta$-lactams and also catalyzes the hydrolysis and aminolysis of small D-alanyl peptides and esters, ${ }^{25}$ although much less efficiently than the R39 enzyme. The general organization of catalytic functional groups in PBP5 is very similar to that in the R39 DD-peptidase, and both resemble that of a class A $\beta$-lactamase; 16 neither, however, contains an analogue of Glu166 of the $\beta$-lactamase - the $\beta$-lactam deacylation catalyst. Extensive structural details of the low-molecular-mass PBPs are provided in a recent review. ${ }^{26}$

The crystal structures described in this article strongly support the predictions made above in regard to DDpeptidase substrate specificity: a specific side-chain binding site is revealed in the R39 DD-peptidase, but not in PBP5.

\section{Results and Discussion}

\section{R39 DD-peptidase structures}

The asymmetric unit of R39 DD-peptidase crystals contains four protein molecules that are not in the same crystallographic environment. ${ }^{16}$ The environment is very similar for monomers $A$ and $D$, and for monomers $B$ and $C$, but is different for both pairs.

In the R39/ 6 complex, all four monomers' active sites are acylated by the ligand, and the conformation of the complex is the same in each monomer. The electron density map calculated in the absence of ligand provides a very clear density showing cephalosporin 6 covalently linked to the enzyme active serine (Fig. 1a). The carbonyl oxygen lies in the oxyanion hole, the carboxylate is oriented towards the hydroxyl group of Thr411 (from the KTG motif), and the amide group of the cephalosporin side chain is wedged between the side chain of Asn300 (from the SXN motif) and the backbone of the strand that lines the active site. In these features, the structure closely resembles those generally found for complexes of $\beta$-lactams with $\beta$-lactam-recognizing enzymes, including that of nitrocefin with the R39 DD-peptidase. ${ }^{16}$

Of particular interest to this article is the disposition of the D- $\alpha$-aminopimelyl side chain. The methylene groups of the side chain are placed between the side chains of Tyr157 and Met414, and the hydrophobic environment is completed by the side chain of Leu349 in the rear of the active site. The side chain of Tyr157 essentially occupies the same position as in the free enzyme; in the nitrocefin complex, it moves to provide hydrophobic cover to the thienyl side chain. ${ }^{16}$ The terminal $\mathrm{H}_{3} \mathrm{~N}^{+}-\mathrm{CH}-\mathrm{COO}^{-}$moiety of the aminopimelic acid is inserted into a pocket composed of residues Trp139 ( $\pi$-cation), Asp142, Arg351, and Ser415 (hydrogen bonds), with a salt bridge between the aminopimelic acid carboxylate and Arg351. The aminopimelyl ammonium-terminus hydrogen bonds to the Asp142 side chain. It is striking that, as with $B$. subtilis PBP4a, ${ }^{18}$ the elements of the side-chain binding site are derived from two separate domains of the protein: ${ }^{16}$ the $\beta$-lactam-binding domain per se and domain II, with the latter supplying Trp139, Asp142, and Tyr147 (Fig. 1a).

In the complex of the enzyme with the peptide $\mathbf{7}$, density maps reveal the presence of $\mathrm{D}-\alpha$-amino- $\varepsilon-$ pimelyl-D-alanine in monomers $\mathrm{A}, \mathrm{B}$, and $\mathrm{C}$ (Fig. 1b). $\mathrm{D}-\alpha$-Amino- $\varepsilon$-pimelyl-D-alanine is one of the products of the carboxypeptidation reaction that is catalyzed by the R39 DD-peptidase. Electron density that could be attributed to the released D-alanine 
(the second product of the reaction) may also have been observed, but we have attributed this electron density to a sulfate ion as in the apostructure of the R39 enzyme. ${ }^{16}$

The D-alanine carboxylate of D- $\alpha$-amino- $\varepsilon$-pimelyl$D$-alanine is turned away from the active-site serine as if it had rotated around the pimelyl $\varepsilon$-carbonpeptide carbonyl bond after the deacylation. A water molecule is present in the oxyanion hole between the active serine and the alanine carbonyl oxygen atoms. Such a rotation was also observed in the complex of the Streptomyces R61 DD-peptidase with a specific peptide product, ${ }^{10}$ but in that case, the carboxylate twisted into the protein, probably by rotation around the D-alanyl $\alpha$-carbon-peptide $\mathrm{N}$ bond, rather than away from the protein as in the present case. Importantly, however, in the present structure, the D- $\alpha$ aminopimelyl side chain is positioned exactly like the side chain of cephalosporin 6 in the $\beta$-lactam complex described above.

The R39 DD-peptidase has a structural homology that is close to that of the B. subtilis PBP4a, which was previously crystallized and soaked with peptide $7 .^{18}$ The positions of the active-site residues of both structures are nearly identical, and the side chain of peptide $\mathbf{7}$ occupies exactly the same position, making identical interactions, particularly with Asp142 and Ser415 (R39 numbering); the one notable difference is that Arg351 is replaced by a histidine in PBP4a. In the primary structure of most PBPs homologous to R39 (e.g., E. coli PBP4 and Neisseria gonorrhoeae PBP3), this residue is a histidine or an arginine, and the other residues forming the meso-diaminopimelate-binding pocket are well conserved. The hydrophobic cleft accommodating the methylene chain is also conserved.

Although monomers A and D have very similar crystallographic environments, the active site of molecule D is not occupied by the ligand. The Asn176 side chain of a symmetry mate inserts into the pocket occupied by the aminopimelyl moiety in the other monomers. In molecule A, the Asn176 side chain (of a symmetric molecule) is clearly shifted, and the pocket is occupied by the side chain of peptide 7 .

\section{PBP5 structures}

In the complex with cephalosporin 6, the acyl linkage with Ser44 and most of the dihydrothiazine ring are clearly defined in the electron density, indicating that cephalosporin 6 has bound and reacted covalently with PBP5 (Fig. 1c). Weak density is observed in the ring around C2. The C-3' acetoxy group is lost, as expected. ${ }^{12,29}$ On moving away from this region, however, less of the molecule is visible. Although some difference peaks that might correspond to the terminal carboxylate and amino groups of the $\mathrm{D}-\alpha$-amino- $\varepsilon$-pimelyl side chain are present near residues Thr217, Asp41, and Leu153, the side chain could not be modeled without ambiguity and thus-extending from the aminopimelyl $\beta$-carbonis excluded from the model. Only a few hydrogenbonding interactions that involve the cephalosporin are present; these hydrogen bonds (none is unexpected on the basis of previous structures of cephem complexes) are between the cephem carboxylate and the hydroxyl group of Thr214 and the side-chain terminus of Arg248 (see below), between the sidechain amide carbonyl and a side-chain NH of Asn112 and the backbone NH of Ser87, and between the acyl-serine carbonyl and the oxyanion hole $(\mathrm{NH}$ of His216 and Ser44). Neither Lys $47 \mathrm{~N}^{\zeta}$ nor Ser110 O ${ }^{\gamma}$ is within the hydrogen-bonding distance of Ser $44 \mathrm{O}^{\gamma}$. This disposition of the latter functional groups, however, is commonly found in complexes of PBPs with $\beta$-lactams and presumably reflects their catalytic incompetence towards deacylation.

A similar picture is observed in the complex of PBP5 with the penicillin 5 (Fig. 1d). The acyl-serine linkage and thiazolidine ring are both clearly visible, except for weak density corresponding to one of the methyl group substituents on the ring (C16). Some density corresponding to the first part of the side chain is visible, but it disappears from the aminopimelyl $\mathrm{C}^{\gamma}$ onwards. Consequently, this region is not included in the model. The enzyme appears to participate in only three hydrogen bonds with the penicillin, between the acyl-serine carbonyl in the oxyanion hole $(\mathrm{NH}$ of His216 and Ser44) and between the side-chain amide $\mathrm{NH}$ and the backbone carbonyl of His216. Notably absent are interactions between the penam carboxylate and the protein: the side chains of neither Thr214 nor Arg248 appear to make contact (cf. the cephalosporin structure described above).

The overall paucity of side-chain electron density for both ligands is an interesting result and suggests that, for PBP5, at least when organized within a crystal lattice, the $\beta$-lactams 5 and 6 can acylate the active-site serine, but beyond the region around the covalent bond, these compounds make relatively few contacts with the enzyme. There is certainly no sign of a specific side-chain binding site as described above for the R39 DD-peptidase. Indeed, this region is

Fig. 1. Crystal structures of the R39 DD-peptidase in complex with (a) the cephalosporin 6 and (b) the peptide 7, and crystal structures of PBP5 from E. coli in complex with (c) the cephalosporin 6 and (d) the penicillin 5. In these stereoviews of the respective active sites, the electron density is a $\left|F_{\mathrm{o}}\right|-\left|F_{\mathrm{c}}\right|$ difference map calculated from the final coordinates of each model refined in the absence of the ligand. The resulting positive density is shown in blue and is contoured at $2.0 \mathrm{\sigma}$. Carbon atoms of each ligand that are visible in the electron density and have been included in the final model are in green. Those that could not be modeled (in PBP5) due to weak density are in gray and are included to show the approximate positions of these groups. The carbon atoms of amino acids that form each active site are in yellow. Oxygen atoms are in red, nitrogen atoms are in blue, and sulfur atoms are in orange. Potential hydrogen bonds are shown as dashed lines, and the distances are noted in angstroms. Some distances beyond hydrogen-bonding range are shown in (d) for comparison with the equivalent distances in (c). This figure was generated using PYMOL (www.pymol.sourceforge.net). 
structured quite differently in PBP5. When compared to the R39 DD-peptidase structure, it comprises only residues from the penicillin-binding domain, whereas

(a)

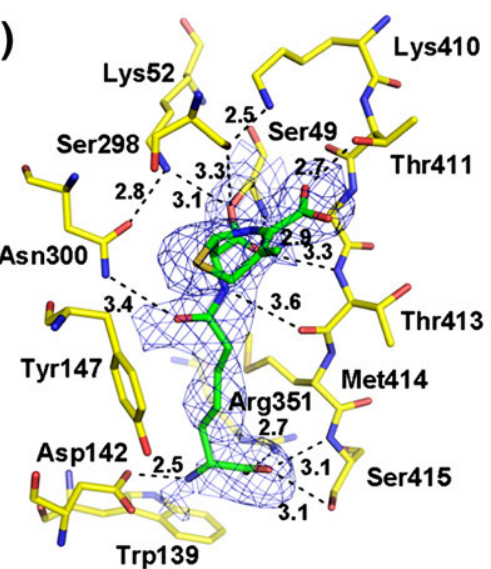

(b)
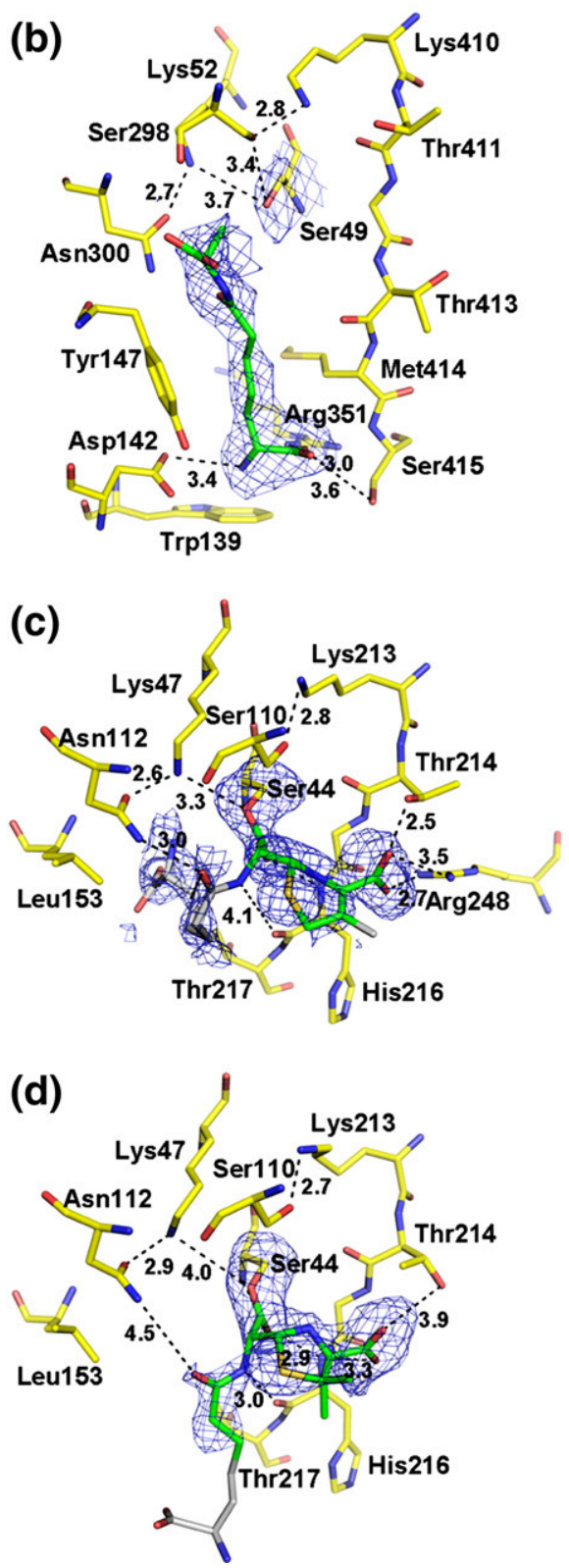

the R39 DD-peptidase includes residues from its domain II, which has no counterpart in PBP5, to bind the peptide terminus.
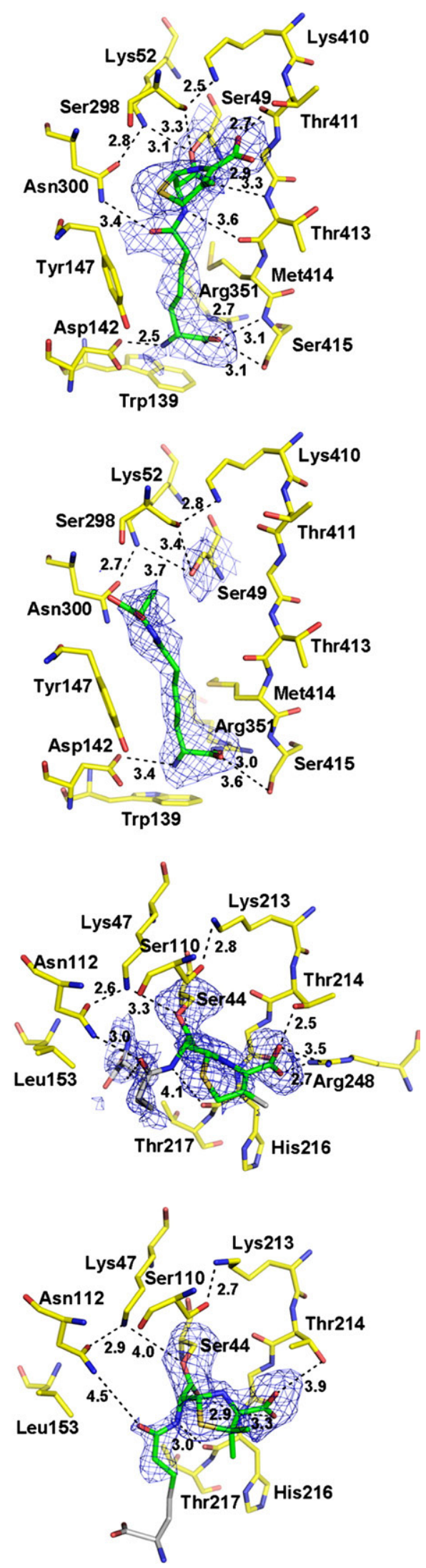

Fig. 1 (legend on previous page) 


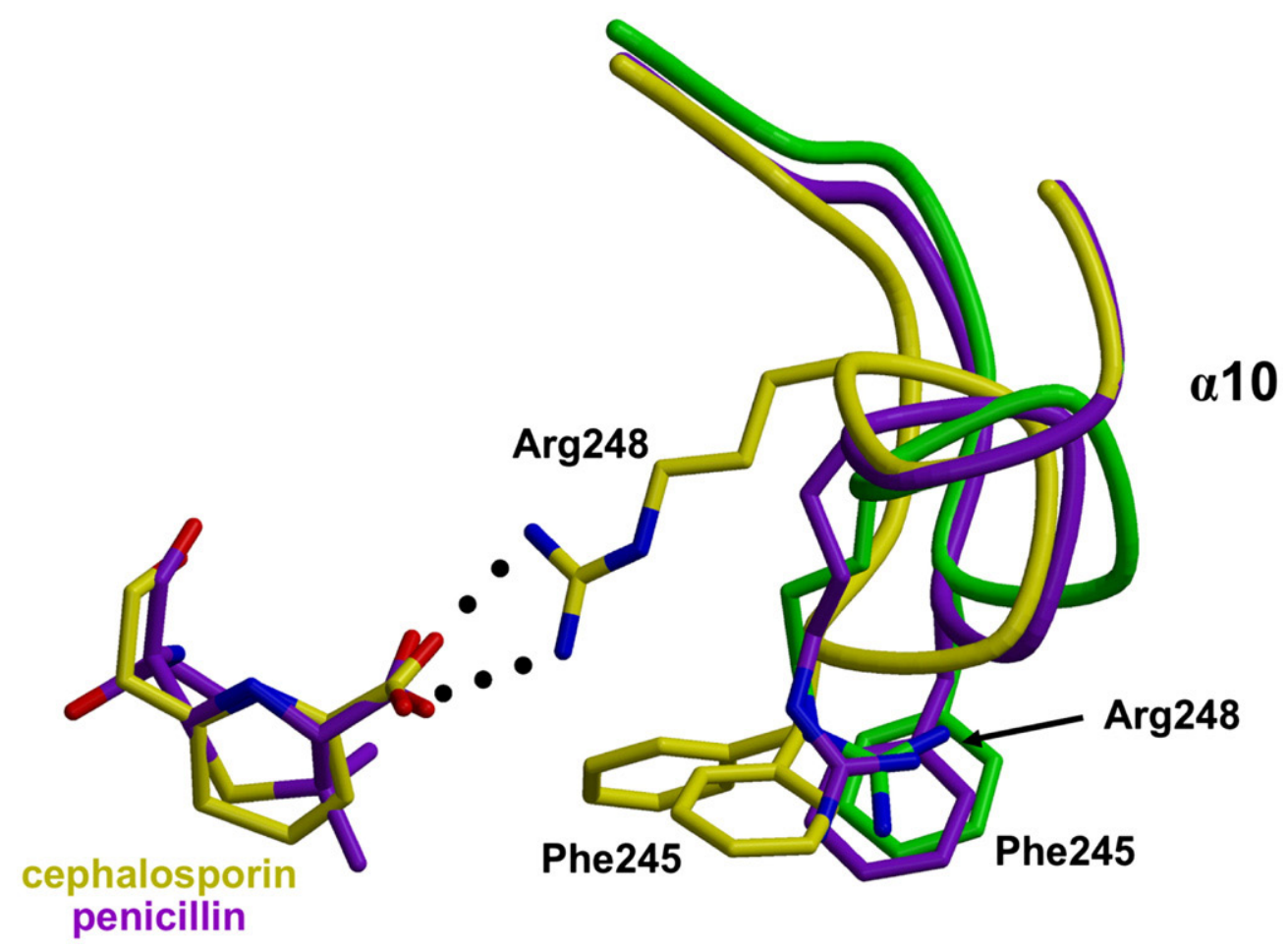

Fig. 2. Interaction between Arg248 and the cephalosporin carboxylate. The backbones of wild-type (green), penicillin5-bound (purple), and cephalosporin-6-bound (yellow) structures of PBP5 are superimposed, showing conformational differences in a loop comprising residues 242-248. In the cephalosporin structure, Arg248 interacts with the carboxylate of the cephalosporin (in yellow), whereas in the penicillin-bound structure, Arg248 occupies a position similar to that in wild-type PBP5. There are also differences in the respective positions of Phe245. The figure was prepared using MOLSCRIPT $^{27}$ and Raster3D. ${ }^{28}$

\section{Comparison with wild-type PBP5}

Both $\beta$-lactam-bound structures were compared with that of the wild-type enzyme. ${ }^{30}$ All main-chain atoms of the cephalosporin-6-bound structure could be superimposed onto the wild-type structure with an RMSD of $0.31 \AA$; the equivalent value for the penicillin-5-bound structure was $0.40 \AA$ A Examination of the superimposed backbones showed them to be virtually indistinguishable, except for one region comprising residues $242-248$, inclusive. In the wildtype enzyme, this region forms a distorted helical region immediately preceding $\alpha 10$, which starts at residue 249 (see Davies et al. ${ }^{31}$ for secondary structure assignments). In the cephalosporin-6-bound structure, however, these residues adopt a more canonical helical conformation such that $\alpha 10$ now starts earlier at residue 245 (Fig. 2). A possible trigger for this conformational change is the interaction between Arg248 and the carboxylate of the cephalosporin. To make this interaction, the guanidinium group of the arginine has shifted by approximately $6 \AA$, when compared to its position in wild-type PBP5. This change in Arg248 appears to destabilize Phe245 because the side chain of this residue now exists in two (and possibly more) conformations. That the carboxylate of the cephalosporin interacts with Arg248 is a little surprising, as we had postulated previously that this group may interact with Arg198, which, in wild-type PBP5, appears much better placed. In fact, the side chain of Arg198 is disordered in the Compound-6-bound structure.

The same region is also slightly altered in the penicillin-5-bound structure, but in this case, the backbone is intermediate in position between wildtype PBP5 and the cephalosporin-6-bound structure (Fig. 2). Again, this region is characterized by relatively weak density. Curiously, the side chain of Arg248 does not interact with penicillin carboxylate and occupies essentially the same position as in the wild-type structure. Thus, there are differences between the interactions with the protein made by the cephalosporin and by the penicillin.

Of further interest, a conformational change that occurred in a complex of PBP5 with a boronic acid peptide mimetic - a shift in residues 152-154 near the active site ${ }^{32}$ - was not observed in either of the ligand-bound structures presented here. Thus, it appears that different compounds reacting with PBP5 induce different and specific conformational changes in the protein (see below).

\section{Discussion}

The structures of complexes of the R39 DD-peptidase with ligands $\mathbf{6}$ and 7, as described above, show very clearly the presence of a specific binding site for the Daminopimelyl side chain (Fig. 1a and b). This result therefore resembles that obtained for the Streptomyces 
R61 DD-peptidase where an analogous side-chain binding site was observed, appropriate for the glycyl-L-aminopimelyl moiety of Streptomyces peptidoglycan. ${ }^{10}$ An analogous site is likely present on $B$. subtilis PBP4a. ${ }^{18}$ In what appears to be sharp contrast, essentially no firm electron density for the side chain, and none particularly for the polar terminus, was observed in the complexes of E. coli PBP5 with $\beta$-lactams 5 and 6 (Fig. 1c and d). This suggests the absence of a specific binding site for the N-terminus of the stem peptide in this enzyme. It is possible that crystal packing interactions may have led to this result with PBP5, but there is certainly no overt indication of this problem such as there is, for example, with monomer D of the R39/peptide 7 complex (see above).

These results are strongly supported by kinetics results. The peptide 7 is an excellent substrate of the R39 DD-peptidase $\left(k_{\mathrm{cat}}=7.4 \mathrm{~s}^{-1} ; K_{\mathrm{m}}=1.3 \mu \mathrm{M} ; k_{\text {cat }} /\right.$ $\left.K_{\mathrm{m}}=5.7 \times 10^{6} \mathrm{~s}^{-1} \mathrm{M}^{-1}\right)^{10}$; similarly, Compounds 5 and 6 are excellent inhibitors. ${ }^{13}$ These numbers mirror those from the analogous peptidoglycan-mimetic peptide 1 with the R61 DD-peptidase $\left(k_{\mathrm{cat}}=69 \mathrm{~s}^{-1}\right.$; $\left.K_{\mathrm{m}}=7.9 \mu \mathrm{M} ; k_{\text {cat }} / K_{\mathrm{m}}=8.7 \times 10^{6} \mathrm{~s}^{-1} \mathrm{M}^{-1}\right)^{6}$ and from analogous $\beta$-lactams. ${ }^{11,13}$ On the other hand, peptide 7 is a very poor substrate of the E. coli PBP5 ( $k_{\text {cat }} /$ $K_{\mathrm{m}}<50 \mathrm{~s}^{-1} \mathrm{M}^{-1}$ ), poorer indeed than the generic peptide $N, N^{\prime}$-diacetyl-L-lysyl-D-alanyl-D-alanine. ${ }^{25}$ The kinetics results published previously and the structures presented here are thus in complete agreement. It should also be noted here that neither the R39 DD-peptidase nor E. coli PBP5 catalyzes hydrolysis of the peptide 3 at rates suggestive of specific interaction. ${ }^{9}$ No strong specificity for this element of peptidoglycan structure has yet been demonstrated for any DD-peptidase. ${ }^{13}$

It seems likely, therefore, that the R39 DD-peptidase has evolved to specifically accept an acyl donor substrate containing the D- $\alpha$-aminopimelyl side chain. The low $K_{\mathrm{m}}$ and therefore high $k_{\text {cat }} / K_{\mathrm{m}}$ values suggest that the substrate is at low effective concentration in vivo. The R39 DD-peptidase is known to catalyze carboxypeptidation, transpeptidation, and endopeptidation reactions in vitro, ${ }^{19,20}$ although its substrate specificity in the latter two reactions has not yet been thoroughly explored. The role of the enzyme in vivo is not known. It may even, as has also been suggested for the Streptomyces R61 DD-peptidase, ${ }^{3,33}$ be a $\beta$-lactam scavenger.

It should be noted, however, that other low-molecular-mass class $C$ enzymes that have structures very similar to those of the R39 enzyme (e.g., E. coli PBP4 and B. subtilis PBP4a $)^{14,17,18}$ do appear to have real, if nonessential (in the same sense as E. coli PBP5 perhaps; see below), roles in bacterial cell wall construction and maintenance. ${ }^{34,35}$ The latter of these enzymes has also been shown to have high hydrolytic activity against the peptide $7 .^{18}$

There is no doubt that E. coli PBP5 does participate in bacterial cell wall construction and/or maintenance. Although it is not essential for cell survival and reproduction, its absence does lead to aberrations in cell shape ${ }^{21}$ and, presumably, a decrease in long-term evolutionary fitness. The structures described above are in agreement with the kinetics results and strongly suggest that no strong binding of peptidoglycanmimetic peptides occurs, to the solubilized enzyme at least. This seems to be true of the membrane-bound holoenzyme also, both in vivo and after isolation in membranes. ${ }^{36}$ It may be that this enzyme recognizes as its specific substrate a much larger segment of peptidoglycan than mimicked by the peptides used in in vitro studies to date. The incorporation of monosaccharides and disaccharides in a complete peptidoglycan monomer did not, however, yield enhanced catalysis. ${ }^{37}$ Alternatively, as has been suggested from time to time, in the two-dimensional milieu of the cell membrane, where the substrate may be effectively immobilized, a high affinity for the substrate may not be required (i.e., sufficient affinity in vivo appears as low affinity in three-dimensional in vitro solution studies). The ease, or otherwise, of physical access of these enzymes to their substrates in vivo is, of course, an unknown factor.

S. pneumoniae PBP3 is another low-molecular-mass class A enzyme that has been studied to some degree both kinetically and structurally. ${ }^{38}$ It appears to be more active in vitro than E. coli PBP5 (the $k_{\text {cat }} / K_{\mathrm{m}}$ for $N, N^{\prime}$-diacetyl-L-lysyl-D-alanyl-D-alanine hydrolysis is $5700 \mathrm{~s}^{-1} \mathrm{M}^{-1}$ ), 38 but still has weak affinity for peptide substrates (the $K_{\mathrm{m}}$ for $N, N^{\prime}$-diacetyl-L-lysylD-alanyl-D-alanine is $19 \mathrm{mM}$; the value of this parameter for E. coli PBP5 is at least this high). ${ }^{25,39}$ This higher activity may reflect the multiple layers of peptidoglycan in Gram-positive bacteria. At any event, it is possible that, even with a weakly specific binding site and correspondingly high $K_{\mathrm{m}}$ values, the $k_{\text {cat }}$ values (e.g., $N, N^{\prime}$-diacetyl-L-lysyl-D-alanyl-Dalanine: for E. coli PBP5 in solution, $k_{\text {cat }}>0.2 \mathrm{~s}^{-1} ; 25,39$ for S. pneumoniae PBP3, $k_{\mathrm{cat}}=110 \mathrm{~s}^{-138}$ ) may be large enough for the in vivo role of these enzymes.

Under either of the above scenarios, the lack of structural specificity towards small molecule substrates is striking and may reflect a powerful defense against small-molecule peptidoglycan-mimetic inhibitors. ${ }^{36}$

It is noticeable in the crystal structures of apo-PBP5 and those of its $\beta$-lactam complexes reported above that the active site appears in a very open conformation, with the active-site residues being more spread out than expected in an active conformation. Simple measures of this are the $C^{\alpha}$ distances from Asn 112 or Ser110 to Gly215. Relevant data are shown in Table 1. Striking from these data are the smaller distances (narrower active-site cleft) in the more reactive $S$. pneumoniae PBP3 and R39 DD-peptidase structures than in PBP5, both alone and in its complexes with $\beta$-lactams 5 and 6 . Striking also are the smaller distances in the PBP5-boronate inhibitor complex where the boronate moiety is hydrogen-bonded to the protein in the manner of a transition-state analogue. ${ }^{32}$ The complexes of PBP5 with $\beta$-lactams 5 and 6 show no such tightening of the structure, which might have been expected to accompany specific recognition. It may be that PBP5, in crystalline form, in solution, and perhaps when resting in vivo, relaxes to an open inactive conformation, unless stimulated by a specific - and perhaps extended- 
Table 1. Active-site distances in DD-peptidases

\begin{tabular}{lcc}
\hline & \multicolumn{2}{c}{ Distance $(\AA)$} \\
\cline { 2 - 3 } Enzyme/ligand & Asn C C Gly C $^{\alpha a}$ & Ser C CGly C $^{\alpha a}$ \\
\hline E. coli PBP5 & 12.7 & 8.9 \\
E. coli PB5/5 & 12.5 & 8.8 \\
E. coli PBP5/6 & 12.4 & 8.7 \\
E. coli PBP5/boronate & 11.8 & 8.1 \\
S. pneumoniae PBP3 & 11.6 & 8.3 \\
R39 DD-peptidase & 11.3 & 8.1 \\
R39 DD-peptidase/6 & 10.8 & 7.8 \\
R39 DD-peptidase/7 & 11.2 & 8.2 \\
\hline
\end{tabular}

a These refer to the Asn and Ser residues of the SXN motif, and to the Gly residue of the KXG motif of the respective enzymes.

substrate. Such a phenomenon could explain its poor reactivity with small substrates, including small peptidoglycan mimetics such as peptide 7. S. pneumoniae PBP3 is more reactive but still not specific for elements of peptidoglycan that are local to the reaction center. ${ }^{13}$ It may be that the two issues, absolute reactivity and substrate specificity, arepartly at least-separable.

The situation described above for E. coli PBP5 and other low-molecular-mass class A enzymes is even more complicated when the essential high-molecularmass enzymes are considered, where little, if any, DDpeptidase activity and little affinity for peptidoglycanmimetic substrates and $\beta$-lactams have been demonstrated in vitro. ${ }^{13}$ The issue of conformational changes necessary for reactivity, both in crystalline form ${ }^{40,41}$ and in solution, ${ }^{42,43}$ has been raised for several of the high-molecular-mass enzymes. As noted above, however, this may relate to absolute reactivity rather than to substrate specificity. With respect to the latter, it would not be surprising if the essential high-molecular-mass DD-peptidases are even more assiduously protected from small substrate-analogue inhibitors than the low-molecular-mass enzymes. It might also be noted here that essentially all PBP structures now available, both free and complexed, display active-site distances very similar to those of S. pneumoniae PBP3 in Table 1. Since these enzymes are certainly not all equally reactive, with peptides for example, the latter distances may be required for optimal activity, but are certainly not sufficient. The exception is PBP2a of Staphylococcus aureus, where the active-site gulf in the crystal form is much narrower-a difference that has been interpreted to reflect the very low reactivity of this $\beta$-lactam-resistant enzyme. ${ }^{40}$

It is clear that much more needs to be done, both structurally and kinetically, for us to understand, qualitatively and quantitatively, how these enzymes function in vivo. That they do function there, however, is even clearer-bacteria certainly flourish, for better and for worse.

\section{Experimental Procedures}

The $\beta$-lactams 5 and 6 and the peptide 7 were obtained as previously described. ${ }^{9,13}$

\section{R39 DD-peptidase crystallography}

The R39 DD-peptidase was expressed and purified as described previously. ${ }^{44}$ The 538 -amino-acid protein precursor presents a 49-amino-acid N-terminal signal peptide and, presumably, a 23-amino-acid C-terminal extension that are both cleaved to yield a mature soluble protein of 466 residues. Crystals were grown at $20{ }^{\circ} \mathrm{C}$ by hanging-drop vapor diffusion. The crystals belong to the space group $P 2_{1}$, with cell dimensions of $a=105.0 \AA, b=93.3 \AA, c=108.2 \AA$, and $\beta=94.2^{\circ}$. The crystallization medium contained protein [2.5 $\mu \mathrm{l}$ of protein solution $\left(18 \mathrm{mg} \mathrm{ml}^{-1}\right)$, also containing $5 \mathrm{mM}$ $\mathrm{MgCl}_{2}$ and $20 \mathrm{mM}$ Tris, $\mathrm{pH}$ 8], $2 \mu \mathrm{l}$ of well solution [2.0 M ammonium sulfate and 0.1 M 2-( $N$-morpholino) ethanesulfonic acid, $\mathrm{pH}$ 6], and $0.5 \mu \mathrm{l}$ of $0.1 \mathrm{M} \mathrm{CoCl}_{2}$ solution. R39 DD-peptidase crystals were transferred to $2 \mu \mathrm{l}$ of well solution containing Compound $6(80 \mathrm{mM})$ for $48 \mathrm{~h}$ to form the R39/6 complex, or to $2 \mu \mathrm{l}$ of well solution containing Compound 7 and D-Ala to final concentrations of $23.4 \mathrm{mM}$ and $900 \mathrm{mM}$, respectively, for $47 \mathrm{~h}$ to form the R39/7 complex. X-ray diffraction experiments were carried out under cryogenic conditions $(100 \mathrm{~K})$ after transferring the crystals into 100\% glycerol. Data were collected with a Rigaku RU-200 rotating anode generator operating at $40 \mathrm{kV}$ and $100 \mathrm{~mA}$, and with a MarResearch Mar345 Imaging Plate $(\lambda=1.5418 \AA)$. Intensities were indexed and integrated using XDS. ${ }^{45}$ Data were scaled with SCALA of the CCP4 program suite, ${ }^{46}$ and all corresponding statistics are given in Table 2.

Using the structure of the apoenzyme, a first round of rigid-body refinement was carried out with REFMAC5. ${ }^{47}$ The resulting unbiased electron density maps displayed in $\operatorname{Coot}^{48}$ unequivocally showed cephalosporin 6 covalently bound to the enzyme in all four monomers and peptide 7 in three monomers. The structure of the R39 DDpeptidase bound to cephalosporin 6 was refined to $2.4 \AA$, and the structure with peptide 7 was refined to $2.25 \AA$. The statistics of refinement are summarized in Table 2.

\section{PBP5 crystallography}

Soluble wild-type PBP5 protein was purified and crystallized as described previously. ${ }^{30}$ These crystals belong to the $C 2$ space group and have cell dimensions of $a=109.4 \AA, b=50.3 \AA, c=84.5 \AA$, and $\beta=120.9^{\circ}{ }^{30}$ Crystal soaking experiments were performed by transferring crystals to stabilizing solutions comprising 8\% polyethylene glycol 400 and $50 \mathrm{mM}$ Tris- $\mathrm{HCl}$ ( $\mathrm{pH} \mathrm{8.0)}$ ) and containing $\beta$-lactam 5 or 6 . Various concentrations of $\beta$-lactams 5 and 6 and soak times were tested. The crystals were then cryoprotected by gradually increasing the concentration of glycerol (in the same solution) in 5\% increments up to $25 \%$ glycerol over a period of about an hour. The degree of incorporation of each compound was assessed by examination of the electron density maps after collection of trial data sets using a rotating anode generator (home $\mathrm{X}$-ray source). The final soaking condition for Compound 5 was $13 \mathrm{mM}$ 
Table 2. Data collection and refinement statistics

\begin{tabular}{|c|c|c|c|c|}
\hline Crystal & $\mathrm{R} 39 / 6$ & $\mathrm{R} 39 / 7$ & $\mathrm{PBP} 5 / 5$ & PBP5/6 \\
\hline \multicolumn{5}{|l|}{ Data collection } \\
\hline Molarity of soak (mM) & 80.0 & 23.4 & 13.2 & 30 \\
\hline Time of soak (h) & 48 & 47 & 1 & 18 \\
\hline Wavelength $(\AA)$ & 1.5418 & 1.5418 & 1.5418 & 0.97934 \\
\hline Resolution range $(\AA)^{\mathrm{a}}$ & $20-2.4(2.53-2.4)$ & $20-2.25(2.37-2.25)$ & $47.0-2.0(2.07-2.00)$ & $41-1.60(1.66-1.60)$ \\
\hline Number of unique reflections & 81,439 & 99,513 & 25,433 & 51,428 \\
\hline$R_{\text {merge }}(\%)^{a, b}$ & $15.0(59.6)$ & $14.7(60.8)$ & $10.7(30.5)$ & $7.4(41.4)$ \\
\hline Redundancy ${ }^{\mathrm{a}}$ & $3.7(3.7)$ & $3.7(3.5)$ & $2.5(2.4)$ & $3.5(2.5)$ \\
\hline Completeness (\%) & $99.8(100)$ & $99.1(94.9)$ & $94.3(85.9)$ & $97.3(82.7)$ \\
\hline$\langle I\rangle /\langle\sigma I\rangle^{\mathrm{a}}$ & $9.6(2.5)$ & $9.7(2.3)$ & $6.8(2.0)$ & $25.7(1.8)$ \\
\hline \multicolumn{5}{|l|}{ Refinement } \\
\hline Resolution range & $16-2.4$ & $15.7-2.25$ & $47.0-2.0$ & $41-1.6$ \\
\hline Number of protein atoms & 13,576 & 13,549 & 2742 & 2738 \\
\hline Number of water molecules & 496 & 600 & 188 & 266 \\
\hline$R_{\text {cryst }}(\%)$ & 20.7 & 23.0 & 23.8 & 19.3 \\
\hline$R_{\text {free }}(\%)$ & 26.0 & 28.5 & 29.6 & 21.5 \\
\hline \multicolumn{5}{|l|}{ RMSD from ideal stereochemistry } \\
\hline Bond lengths $(\AA)$ & 0.009 & 0.010 & 0.011 & 0.011 \\
\hline Bond angles $\left({ }^{\circ}\right)$ & 1.22 & 1.28 & 1.40 & 1.29 \\
\hline \multicolumn{5}{|l|}{$B$-factors } \\
\hline Mean $B$-factor (all atoms) $\left(\AA^{2}\right)$ & 28.9 & 26.4 & 28.0 & 21.3 \\
\hline Mean $B$-factor (main chain) $\left(\AA^{2}\right)$ & 28.7 & 26.2 & 27.4 & 18.9 \\
\hline RMSD in main-chain $B$-factors $\left(\AA^{2}\right)$ & 0.53 & 0.32 & 0.53 & 0.59 \\
\hline $\begin{array}{l}\text { Mean } B \text {-factor (side chains and water } \\
\text { molecules) }\left(\AA^{2}\right)\end{array}$ & 29.1 & 26.7 & 28.6 & 23.5 \\
\hline RMSD in side-chain $B$-factors $\left(\AA^{2}\right)$ & 1.19 & 0.70 & 1.13 & 1.40 \\
\hline \multicolumn{5}{|l|}{ Ramachandran plot } \\
\hline Most favored region (\%) & 88.4 & 90.0 & 92.2 & 92.5 \\
\hline Additionally allowed regions (\%) & 10.8 & 9.4 & 6.9 & 7.2 \\
\hline Generously allowed regions (\%) & 0.6 & 0.5 & 0.7 & 0.3 \\
\hline Disallowed regions (\%) & 0.1 & 0.1 & 0.3 & 0.0 \\
\hline RMSD of $C^{\alpha}$ atom with native structure $(\AA)$ & $0.43^{c}$ & 0.48 & 0.40 & 0.31 \\
\hline
\end{tabular}

for $1 \mathrm{~h}$, and that for cephalosporin 6 was $30 \mathrm{mM}$ for $18 \mathrm{~h}$.

For the complex with penicillin 5, data were collected on an R-AXIS IV ${ }^{++}$image plate detector mounted on an RU-H3R rotating anode X-ray generator fitted with Osmic Confocal Optics (Rigaku MSC). Data $\left(180^{\circ}\right)$ were collected and processed with $\mathrm{d}^{*}$ Trek. ${ }^{49}$

Data for the complex with cephalosporin 6 were collected at the SER-CAT ID22 beamline of the Advanced Photon Source on a Mar 300 CCD detector at a wavelength of $0.979 \AA$. The data were processed using HKL2000. ${ }^{50}$

In both cases, the starting structure for refinement was the structure of wild-type PBP $5,{ }^{30}$ but stripped of water molecules and other ligands. After an initial round of refinement, using $\mathrm{CNS}^{51}$ for cephalosporin 6 or using REFMAC $5^{47}$ for Compound 5 , a difference electron density map $\left(\left|F_{\text {obs }}\right|-\left|F_{\text {calc }}\right|\right)$ was calculated and displayed using the graphics program O. ${ }^{52}$ The coordinates for each compound were built in the Monomer Library Sketcher module of REFMAC5 and manually positioned into the unbiased positive electron density in the active site of the enzyme using $\mathrm{O}$. Thereinafter, each model was refined using REFMAC5, alternating with rounds of manual revision. A subset (5\%) of the reflections was set aside for calculation of the free $R$-factor. ${ }^{53}$ Water molecules with reasonable hydrogen bond distances were included in later rounds of refinement, and several amino acid side chains were modeled with multiple conformations. The stereochemistries of the final models were evaluated using PROCHECK. ${ }^{54}$ The structure, in complex with the cephalosporin 6, was refined to $1.6 \AA$ resolution with an $R$-factor of $21.2 \%$ and an $R_{\text {free }}$ of $23.5 \%$, and that with the penicillin 5 was refined at $2.0 \AA$ with an $R$-factor of $23.8 \%$ and an $R_{\text {free }}$ of $29.6 \%$. Both structures have excellent stereochemistry (see Table 2).

\section{Protein Data Bank accession codes}

The atomic coordinates for the crystal structures described above have been deposited in the RCSB Protein Data Bank and are available under accession codes 2VGJ (R39/6 complex), 2VGK (R39/7 complex), 3BEB (PBP5/5), and 3BEC (PBP5/ 6 complex).

\section{Acknowledgements}

Use of the Advanced Photon Source for the PBP5 project (A.J.P., J.H., and C.D.) was supported by the 
US Department of Energy, Office of Science, Office of Basic Energy Sciences, under contract no. W-31109-ENG-38. Data were collected at the Southeast Regional Collaborative Access Team BM22 beamline at the Advanced Photon Source, Argonne National Laboratory. Supporting institutions may be found at www.ser-cat.org/members.html. The X-ray crystallography facility used for the PBP5 work was supported by the Medical University of South Carolina's Research Resource Facilities program.

The R39 project (E.S. and P.C.) was supported, in part, by European Commission Sixth Framework Program grants LSMH-CT-COBRA 2003-503335 and LSMH-CT-EUR-INTAFAR 2004-512138; by the Belgian Program on Interuniversity Poles of Attraction initiated by the Belgian State, Prime Minister's Office, Science Policy programming (IAP no. P6/19); by the Actions de Recherche Concertées (grant 03/08-297); by the Fonds National de la Recherche Scientifique (IISN 4.4505.00, FRFC 9.45/9.99, FRFC 2.4.508.01.F, FRFC 9.4.538.03.F, and FRFC 2.4.524.03); and by the University of Liège (Fonds spéciaux, Crédit classique, 1999). This research was also supported by National Institutes of Health grants AI-17986 (R.F.P.) and GM66861 (C.D.).

\section{Author Contributions}

ES and PC carried out the crystallographic analyses on R39. AJP, JH and CD carried out the crystallographic analyses on PBP5. HRJ and RFP performed the syntheses and kinetics analysis. ES, CD and RFP wrote the paper.

\section{References}

1. Macheboeuf, P., Contreras-Martell, C., Job, V., Dideberg, O. \& Dessen, A. (2006). Penicillin-binding proteins: key players in bacterial cell cycle and drug resistance processes. FEMS Microbiol. Rev. 30, 673-691.

2. Ghuysen, J.-M. (1991). Serine $\beta$-lactamases and penicillin-binding proteins. Annu. Rev. Microbiol. 45, 37-67.

3. Goffin, C. \& Ghuysen, J.-M. (2002). Biochemistry and comparative genomics of SxxK superfamily acyltransferases offer a clue to the mycobacterial paradox: presence of penicillin-susceptible target proteins versus lack of efficiency of penicillin as a therapeutic agent. Microbiol. Mol. Biol. Rev. 66, 702-738.

4. Ghuysen, J.-M., Frère, J.-M., Leyh-Bouille, M., Coyette, J., Dusart, J. \& Nguyen-Distèche, M. (1979). Use of model enzymes in the determination of the mode of action of penicillins and $\Delta^{3}$-cephalosporins. Annu. Rev. Biochem. 48, 73-101.

5. Pratt, R. F. (2008). Substrate specificity of bacterial DDpeptidases (penicillin-binding proteins). Cell. Mol. Life Sci., in press (DOI 10.1007/s00018-008-7591-7).

6. Anderson, J. W. \& Pratt, R. F. (2000). Dipeptide binding to the extended active site of the Streptomyces R61 D-alanyl-D-alanine peptidase: the path to a specific substrate. Biochemistry, 39, 12200-12209.

7. Kumar, I. \& Pratt, R. F. (2005). Transpeptidation reactions of a specific substrate catalyzed by the Streptomyces R61 DD-peptidase: characterization of a chromogenic substrate and acyl acceptor. Biochemistry, 44, 9971-9979.
8. Kumar, I. \& Pratt, R. F. (2005). Transpeptidation reactions of a specific substrate catalyzed by the Streptomyces R61 DD-peptidase: the structural basis of acyl acceptor specificity. Biochemistry, 44, 9961-9970.

9. Anderson, J. W., Adediran, S. A., Charlier, P., NguyenDistèche, M., Frère, J.-M., Nicholas, R. A. \& Pratt, R. F. (2003). On the substrate specificity of bacterial DDpeptidases: evidence from two series of peptidoglycan-mimetic peptides. Biochem. J. 373, 949-955.

10. McDonough, M. A., Anderson, J. W., Silvaggi, N. R., Pratt, R. F., Knox, J. R. \& Kelly, J. A. (2002). Structures of two kinetic intermediates reveal species specificity of penicillin-binding proteins. J. Mol. Biol. 322, 111-122.

11. Josephine, H. R., Kumar, I. \& Pratt, R. F. (2004). The perfect penicillin? Inhibition of a bacterial DD-peptidase by peptidoglycan-mimetic $\beta$-lactams. J. Am. Chem. Soc. 126, 8122-8123.

12. Silvaggi, N., Josephine, H. R., Kuzin, A. P., Nagarajan, R., Pratt, R. F. \& Kelly, J. A. (2005). Crystal structures of complexes between the R61 DD-peptidase and peptidoglycan-mimetic $\beta$-lactams: a non-covalent complex with a "perfect penicillin". J. Mol. Biol. 345, 521-533.

13. Josephine, H. R., Charlier, P., Davies, C., Nicholas, R. A. \& Pratt, R. F. (2006). Reactivity of penicillinbinding proteins with peptidoglycan-mimetic $\beta$-lactams: what's wrong with these enzymes? Biochemistry, 45, 15873-15883.

14. Granier, B., Duez, C., Lepage, S., Englebert, S., Dusart, J., Dideberg, O. et al. (1992). Primary and predicted secondary structures of the Actinomadura R39 extracellular DD-peptidase, a penicillin-binding protein (PBP) related to Escherichia coli PBP4. Biochem. J. 282, 781-788.

15. Duez, C., Vanhove, M., Gallet, X., Bouillenne, F., Docquier, J.-D., Brans, A. \& Frère, J.-M. (2001). Purification and characterization of PBP4a, a new lowmolecular-weight penicillin-binding protein from Bacillus subtilis. J. Bacteriol. 183, 1595-1599.

16. Sauvage, E., Herman, R., Petrella, S., Duez, C., Bouillenne, F., Frère, J.-M. \& Charlier, P. (2005). Crystal structure of the Actinomadura R39 DD-peptidase reveals new domains in penicillin-binding proteins. J. Biol. Chem. 280, 31249-31256.

17. Kishida, H., Unzai, S., Roper, D. I., Lloyd, A., Park, S.-Y. \& Tame, J. R. H. (2006). Crystal structure of penicillin-binding protein 4 (dacB) from Escherichia coli, both in the native form and covalently linked to various antibiotics. Biochemistry, 45, 783-792.

18. Sauvage, E., Duez, C., Herman, R., Kerff, F., Perrella, S., Anderson, J. W. et al. (2007). Crystal structure of the Bacillus subtilis penicillin-binding protein $4 \mathrm{a}$, and its complex with a peptidoglycan-mimetic peptide. J. Mol. Biol. 371, 528-539.

19. Ghuysen, J.-M., Leyh-Bouille, M., Campbell, J. N., Moreno, R., Frère, J.-M., Duez, C. et al. (1973). Structure of the wall peptidoglycan of Streptomyces R39 and the specificity profile of its exocellular DDcarboxypeptidase-transpeptidase for peptide acceptors. Biochemistry, 12, 1243-1251.

20. Zhao, G.-H., Duez, C., Lepage, S., Forceille, C., Rhazi, N., Klein, D. et al. (1997). Site-directed mutagenesis of the Actinomadura R39 DD-peptidase. Biochem J. 327, 377-381.

21. Nelson, D. E. \& Young, K. D. (2000). Penicillin-binding protein 5 affects cell diameter, contour and morphology of Escherichia coli. J. Bacteriol. 182, 1714-1721.

22. Matsuhashi, M., Tamaki, S., Curtis, S. J. \& Strominger, J. L. (1979). Mutational evidence for identity of penicillin-binding protein 5 in Escherichia coli with the major D-alanine carboxypeptidase IA activity. J. Bacteriol. 137, 644-647. 
23. Harris, F., Brandenburg, K., Seydel, U. \& Phoenix, D. (2002). Investigations into the mechanisms used by the C-terminal anchors of Escherichia coli penicillinbinding proteins $4,5,6$ and $6 \mathrm{~b}$ for membrane interactions. Eur. J. Biochem. 269, 5821-5829.

24. Nicholas, R. A., Krings, S., Tomberg, J., Nicola, G. \& Davies, C. (2003). Crystal structure of wild-type penicillin-binding protein 5 from Escherichia coli. J. Biol. Chem. 278, 644-647.

25. Stefanova, M. E., Davies, C., Nicholas, R. A. \& Gutheil, W. G. (2002). pH, inhibitor and substrate specificity studies on Escherichia coli penicillin-binding protein 5. Biochim. Biophys. Acta, 1597, 292-300.

26. Sauvage, E., Kerff, F., Terrak, M., Ayala, J. A. \& Charlier, P. (2008). The penicillin-binding proteins: structure and role in peptidoglycan biosynthesis. FEMS Microbiol. Rev. 32, 234-258.

27. Kraulis, P. J. (1991). MOLSCRIPT: a program to produce both detailed and schematic plots of protein structures. J. Appl. Crystallogr. 24, 946-950.

28. Merritt, E. A. \& Murphy, M. E. P. (1994). Raster3D version 2.0. A program for photorealistic molecular graphics. Acta Crystallogr. Sect. D, 50, 869-873.

29. Faraci, W. S. \& Pratt, R. F. (1986). Interactions of cephalosporins with the Streptomyces R61 DD-transpeptidase/carboxypeptidase. Influence of the 3'-substituent. Biochem. J. 238, 309-312.

30. Nicholas, R. A., Krings, S., Tomberg, J., Nicola, G. \& Davies, C. (2003). Crystal structure of wild-type penicillin binding protein 5 from E. coli: implications for deacylation of the acyl-enzyme complex. J. Biol. Chem. 278, 52826-52833.

31. Davies, C., White, S. W. \& Nicholas, R. A. (2001). Crystal structure of a deacylation-defective mutant of penicillin-binding protein 5 at $2.3 \AA$ resolution. J. Biol. Chem. 276, 616-623.

32. Nicola, G., Peddi, S., Stefanova, M., Nicholas, R. A., Gutheil, W. G. \& Davies, C. (2005). Crystal structure of Escherichia coli penicillin-binding protein 5 bound to a tripeptide boronic acid inhibitor: a role for Ser-110 in deacylation. Biochemistry, 44, 8207-8217.

33. Kelly, J. A., Dideberg, O., Charlier, P., Wery, J. P., Libert, M., Moews, P. C. et al. (1986). On the origin of bacterialresistance to penicillin-comparison of a beta-lactamase and a penicillin target. Science, 231, 1429-1431.

34. Priyadarshini, R., Popham, D. L. \& Young, K. D. (2006). Daughter cell separation by penicillin-binding proteins and peptidoglycan amidases in Escherichia coli. J. Bacteriol. 188, 5345-5355.

35. Scheffers, D.-J., Jones, L. J. \& Errington, J. (2004). Several distinct localization patterns for penicillin-binding proteins in Bacillus subtilis. Mol. Microbiol. 51, 749-764.

36. Kumar, I., Josephine, H. R. \& Pratt, R. F. (2007) Reactions of peptidoglycan-mimetic $\beta$-lactams with penicillin-binding proteins in vivo and in membranes. ACS Chem. Biol. 2, 620-624.

37. Hesek, D., Suvarov, M., Morio, K., Lee, M., Brown, S. Vakulenko, S. B. \& Mobashery, S. (2004). Synthetic peptidoglycan substrates for penicillin-binding proteins of Gram-negative bacteria. J. Org. Chem. 69, 778-784.

38. Morlot, C., Pernot, L., Le Gouellec, A., Di Giulmi, A. M., Vernet, T., Dideberg, O. \& Dessen, A. (2005). Crystal structures of a peptidoglycan synthesis regulatory factor (PBP3) from Streptococcus pneumoniae. J. Biol. Chem. 280, 15984-15991.
39. Zhang, W., Shi, Q., Meroueh, S. O., Vakulenko, S. B. \& Mobashery, S. (2007). Catalytic mechanism of penicillin-binding protein 5 of Escherichia coli. Biochemistry, 46, 10113-10121.

40. Lim, D. \& Strynadka, N. C. J. (2002). Structural basis for the $\beta$-lactam resistance of PBP 2a from methicillinresistant Staphylococcus aureus. Nat. Struct. Biol. 9, 870-876.

41. Macheboeuf, P., Di Giulmi, A. M., Job, V., Vernet, T., Dideberg, O. \& Dessen, A. (2005). Active site restructuring regulates ligand recognition in class $\mathrm{A}$ penicillin-binding proteins. Proc. Natl Acad. Sci. USA, 102, 577-582.

42. Fuda, C., Hesek, D., Lee, M., Morio, K.-I., Nowak, T. \& Mobashery, S. (2005). Activation for catalysis of penicillin-binding protein 2a from methicillin-resistant Staphylococcus aureus by bacterial cell wall. J. Am. Chem. Soc. 127, 2056-2057.

43. Fuda, C., Hesek, D., Lee, M., Heilmayer, W., Novak, R., Vakulenko, S. B. et al. (2006). Mechanistic basis for the action of new cephalosporin antibiotics effective against methicillin- and vancomycin-resistant Staphylococcus aureus. J. Biol. Chem. 281, 10035-10041.

44. Granier, B., Duez, C., Lepage, S., Englebert, S., Dusart, J., Dideberg, O. et al. (1992). Primary and predicted secondary structures of the Actinomadura R39 extracellular DD-peptidase, a penicillin-binding protein (PBP) related to the Escherichia coli PBP4. Biochem. J. 282, 781-788.

45. Kabsch, W. (1993). Automatic processing of rotation diffraction data from crystals of initially unknown symmetry and cell constants. J. Appl. Crystallogr. 26, 795-800.

46. CCP4 (1994). The CCP4 suite: programs for protein crystallography. Acta Crystallogr. Sect. D, 50, 760-763.

47. Murshudov, G. N., Vagin, A. A. \& Dodson, E. J. (1997). Refinement of macromolecular structures by the maximum-likelihood method. Acta Crystallogr. Sect. $D, 53,240-255$.

48. Emsley, P. \& Cowtan, K. (2004). Coot: model-building tools for molecular graphics. Acta Crystallogr. Sect. D, 60, 2126-2132.

49. Pflugrath, J. W. (1999). The finer things in X-ray diffraction data collection. Acta Crystallogr. Sect. D, 55, 1718-1725.

50. Otwinowski, Z. \& Minor, W. (1997). Processing of $X$-ray diffraction data collected in oscillation mode. Methods Enzymol. 276, 307-326.

51. Brünger, A. T., Adams, P. D., Clore, G. M., De Lano, W. L., Gros, P., Grosse-Kunstleve, R. W. et al. (1998). Crystallography and NMR system: a new software suite for macromolecular structure determination. Acta Crystallogr. Sect. D, 54, 905-921.

52. Jones, T. A., Zou, J. Y., Cowan, S. W. \& Kjeldgaard, M. (1991). Improved methods for building protein structures in electron-density maps and the location of errors in these models. Acta Crystallogr. Sect. A, 47, 110-119.

53. Brünger, A. T. (1992). Free $R$ value: a novel statistical quantity for assessing the accuracy of crystal structures. Nature, 355, 472-474.

54. Laskowski, R. A., MacArthur, M. W., Moss, D. S. \& Thornton, J. M. (1993). PROCHECK: a program to check the stereochemical quality of protein structures. J. Appl. Crystallogr. 26, 283-291. 